\section{MS7-P2 Klf4 and Grhl1: Two distinct ways of achieving DNA target site recognition}

Udo Heinemann ${ }^{1}$, Qianqian Ming ${ }^{1}$, Yvette Roske ${ }^{1}$, Anja Schütz ${ }^{1}$

1. Max Delbrück Center for Molecular Medicine, Berlin

email: heinemann@mdc-berlin.de

Cell- and tissue-specific gene expression is controlled at the transcriptional and translational levels. Transcription factors bind to target sites on double-stranded DNA that are characterized by distinct nucleotide sequence motifs. Recently, we have studied two human transcription factors, Klf4 and Grhl1, that appear to utilize different molecular strategies for achieving target site recognition.

Krueppel-like factor-4 (Klf4) belongs to a family of zinc-finger transcription factors and is indispensable for the terminal maturation of epithelial tissues (1). In combination with the transcription factors Sox2, Oct4 and c-Myc, Klf4 has the potential to revert differentiated cells to a pluripotent cell-like state (2). The crystal structure of the Klf4 zinc finger region bound to target DNA shows the arrangement of three $\mathrm{CCHH}$ zinc fingers in the DNA major groove and the formation of multiple DNA sequence specific hydrogen bonds between protein side chains and DNA base pairs (3)

Mammalian Grainyhead-like-1 (Grhl1) is grouped within a family of transcription factors along with Grhl2, Grhl3 and the CP2 factors for which no structural information was available until recently. These protein play important roles in early ambryogenesis, have documented functions in adult mice and are linked to human disease $(4,5)$. The crystal structure of the DNA-binding domain of human Grhl1 (Grhl1 DBD) unexpectedly revealed a fold closely resembling the central tumor suppressor p53. Subsequent structure analysis of the DNA target site-bound Grhl1 DBD showed a mode of DNA sequence readout that relies on a small number of direct hydrogen bonds between the protein and DNA bases and additional contacts to the DNA backbone. This binding mode is fully supported by DNA binding assays using mutated Grhll variants and DNA target sites.

References

1. Shields, J.M. \& Yang,, V.W. (1998) Nucleic Acids Res. 26, 796

2. Takahashi, K., Tanabe, K., Ohnuki, M., Narita, M., Ichisaka, T., Tomoda, K. \& Yamanaka, S. (2007) Cell 131,861 .

3. Schuetz, A., Nana, D., Rose, C., Zocher, G., Milanovic, M., Koenigsmann, J., Blasig, R., Heinemann, U. \& Carstanjen, D. (2011) Cell. Mol. Life Sci. 68, 3121.

4. Rifat, Y., Parekh, V., Wilanowski, T., Hislop, N.R., Auden, A., Ting, S.B. \& Jane, S.M. (2010) Dev. Biol. 345, 237.

5. Wang, S. \& Samakovlis, C. (2012) Curr. Topics Dev. Biol. 98, 35 .

Keywords: Transcription factors, Krueppel-like factor-4 (Klf4), Grainyhead-like-1 (Grhl1), protein-DNA binding, DNA sequence recognition
MS7-P3 Structure of the EnvZ periplasmic domain with CHAPS reveals the mechanism of porin inactivation by bile salts Eunha Hwang ${ }^{1}$, Hae-Kap Cheong ${ }^{1}$, Young Ho Jeon ${ }^{2}$, Chaejoon
Cheong

1. Division of Bioconvergence Analysis, Korea Basic Science Institute (KBSI), Ochang, Chungbuk, Republic of Korea

2. College of Pharmacy, Korea University, Sejong, Republic of Korea

email: hwang0131@kbsi.re.kr

The EnvZ-OmpR two-component system senses external osmolarity and responds to environmental signals through the regulation of the outer membrane porins $\mathrm{OmpF}$ and OmpC. Although extensive studies for the EnvZ signaling have been reported, the structure of the periplasmic sensor domain at atomic resolution is not available yet. Here, we present the first crystal structure of the EnvZ periplasmic domain (EnvZ-PD) with CHAPS. The asymmetric unit contains a single monomer of EnvZ-PD and two molecules of CHAPS. The structure of EnvZ-PD showed similar folding topology to the PDC domains of PhoQ, DcuS, and CitA, but distinct orientations of helices and $\beta$-hairpin structures. Size exclusion chromatography and cross-linking experiment suggest that EnvZ-PD is in monomer-dimer equilibrium state in solution. It is likely that the CHAPS molecules hinder the dimerization of EnvZ-PD by changing the orientation of $\beta$-hairpin. When CHAPS is replaced by sodium cholate, a major component of bile salts, the overall folding of EnvZ-PD is retained. In vivo $\beta$-galactosidase assay showed that ompC-lacZ gene expression was dose-dependently inhibited by the addition of CHAPS and sodium cholate. These results provide an insight into the inactivation mechanism of porins in the EnvZ/OmpR signaling pathway, which is caused by the presence of bile salts.

Keywords: EnvZ 